\title{
Automatic Recognition of Cortical Sulci Using a Congregation of Neural Networks
}

\author{
Denis Rivière $^{1}$, Jean-François Mangin ${ }^{1}$, Dimitri Papadopoulos-Orfanos ${ }^{1}$, \\ Jean-Marc Martinez ${ }^{2}$, Vincent Frouin ${ }^{1}$, and Jean Régis ${ }^{3}$ \\ 1 Service Hospitalier Frédéric Joliot, CEA, 91401 Orsay, France \\ mangin@shfj.cea.fr, http://www-dsv.cea.fr/ \\ 2 Service d'Etude des Réacteurs et de Mathématiques Appliquées, CEA, Saclay \\ 3 Service de Neurochirurgie Fonctionnelle et Stéréotaxique, La Timone, Marseille
}

\begin{abstract}
This paper describes a complete system allowing automatic recognition of the main sulci of the human cortex. This system relies on a preprocessing of MR images leading to abstract structural representations of the cortical folding. The representation nodes are cortical folds, which are given a sulcus name by a contextual pattern recognition method. This method can be interpreted as a graph matching approach, which is driven by the minimization of a global function made up of local potentials. Each potential is a measure of the likelihood of the labelling of a restricted area. This potential is given by a multi-layer perceptron trained on a learning database. A base of 26 brains manually labelled by a neuroanatomist is used to validate our approach. The whole system developed for the right hemisphere is made up of 265 neural networks.
\end{abstract}

\section{Introduction}

The development of image analysis methods dedicated to automatic management of brain anatomy is a widely addressed area of research. A number of works focus on the notion of deformable atlases, which can be elastically transformed to reflect the anatomy of new subjects. An exhaustive bibliography of this approach initially proposed by Bajcsy [1] is largely beyond the scope of this paper (see [23] for a recent review). The complexity and the striking interindividual variability of the human cortex folding patterns, however, have led several groups to question the behaviour of the deformable atlas framework at the cortex level. The main issues to be addressed are the following:

What are the features of the cortex folding patterns which should be matched across individuals? While some sulci clearly belong to this set of landmark features because they are usually considered as boundaries between different functional areas, nobody knows to which extent secondary folds should play the same role [18]. Some answers to this important issue could stem from foreseeable advances in functional imaging and mapping of cortex connectivity. Deformable atlas methods rely on the optimization of some function which realizes a trade-off between similarity to the new brain and deformation cost. Whatever the approach, the function driving the deformations is non convex. When 
high-dimensional deformation fields are used, this non-convexity turns out to be particularly problematic since standard optimization approaches are bound to lead to a local optimum. While multi-resolution methods may guarantee that an "interesting optimum" is found, the complexity of the cortical folding patterns implies that a lot of other similar optima exist. An important issue is raised by this observation: is the global optimum the best one according to the pairing of sulcal landmarks?

To overcome some of the difficulties related to the non-convexity of the problem, several teams have proposed to design composite similarity functions relying on manual identifications of the main sulci $[23,4]$. These composite functions impose the pairing of homologous sulcal landmarks. While a lot of work remains to be done along this line, this evolution seems required to adapt the deformable atlas paradigm to the human cortex. This new point of view implies a preprocessing of the data in order to extract and identify automatically these sulcal landmarks, which is the subject of our paper.

Our system may be considered as a symbolic version of the deformable atlas approach. The framework is made up of two stages [14, 15]. An abstract structural representation of the cortical topography is extracted first from each new T1-weighted MR image. A symbolic pattern recognition method is then used to label automatically the main sulci. This method can be interpreted as a graph matching approach. Hence the usual iconic anatomical template is replaced by an abstract structural template. The one to many matching between the template nodes and the nodes of one structural representation is simply a labelling operation. This labelling is driven by the minimization of a global function made up of local potentials. Each local potential is a measure of the likelihood of the labelling of a restricted cortex area. This potential is given by a virtual expert in this area made up of a multi-layer perceptron trained on a learning database.

While the complexity of the preprocessing stage required by our method may appear as a weakness compared to the straightforward use of continuous deformations, it results in a fundamental difference. While the evaluation of functions driving continuous deformations is costly in terms of computation, the function used to drive the symbolic recognition relies on only a few hundred labels and can be evaluated at a low cost. Hence stochastic optimization algorithms can be used to deal with the non-convexity problems. In fact, working at a higher level of representation leads to more efficiency for the pattern recognition process, which explains an increasing interest in the community $[10,8,9]$.

\section{The Preprocessing Stage}

This section describes briefly the robust sequence of treatments that converts automatically a T1-weighted MR image in an abstract structural representation of the cortical topography. The whole sequence requires about one hour on a conventional workstation, including sophisticated triangulation of hundreds of objects. All the steps have been validated with at least 50 different images acquired with 6 different scanners using various MR sequence parameters. The processing sequence is the following: 
1. Bias Correction via minimization of the grey level distribution entropy [12];

2. Histogram scale space analysis to assess tissue statistics [13];

3. Brain segmentation using mathematical morphology and Markov fields [13];

4. Hemisphere separation using mathematic morphology [16];

5. Segmentation of grey/CSF using topology preserving deformations [14];

6. Homotopic skeletonization driven by an isophote based erosion [14];

7. Segmentation of the skeleton into topologically simple surfaces [14];

8. Simple surfaces are split according to fold depth local minima, which are considered as clues of a putative burried gyrus [18, 14, 11, 10];

9. The objects provided by the last step are finally gathered in a structural representation which describes their relationships. Three kinds of links are created between these nodes: $\rho_{T}$ links represent skeleton splits related to the simple surface definition; $\rho_{P}$ links represent splits related to the presence of a putative burried gyrus; and $\rho_{C}$ links represent a neighborhood relationship geodesic to the hemisphere hull [14]. The resulting graph is enriched with numerous semantic attributes dedicated to the recognition stage. Nodes are described by their size, maximal depth, gravity center localization, and mean normal. Links of type $\rho_{T}$ and $\rho_{P}$ are described by their length, extremity localizations, minimal and maximal depth, and mean direction. Links of type $\rho_{C}$ are described by their size and the localization of the closest points of the linked nodes. The resulting attributed graph is supposed to include all the information required by the sulcus recognition process.

\section{The Learning Database}

Our preprocessing tool can be viewed as a compression system which provides for each individual brain a synthetic description of the cortex folding patterns. A sophisticated 3D browser allows our neuroanatomist to label manually each node with a name chosen in a list of anatomical entities. The lack of a validated explanation of the structural variability of the human cortex is an important problem during this labelling. Indeed, standard sulci are often split into several pieces which leads to ambiguous configurations [17].

It has to be understood that this situation prevents the definition of an unquestionable gold standard to be reached by any sulcus recognition method. Therefore, one of the aims of our research is to favour the emergence of new anatomical descriptions relying on smaller sulcal entities than the usual ones. According to different arguments that would be too long to develop in this paper, these units, the primary cortical folds that appear on the foetal cortex, are stable across individuals; a functional delimitation meaning is probably attached to them [18]. During ulterior stages of brain growth, some of these sulcal roots merge with each other and form different patterns depending on the subjects. The more usual patterns correspond to the usual sulci. In our opinion, some clues on these sulcal root fusions can be found in the depth of the sulci.

A model of these sulcal roots derived from our anatomical research has been used to label 26 right hemispheres. This model shares striking similarities with 

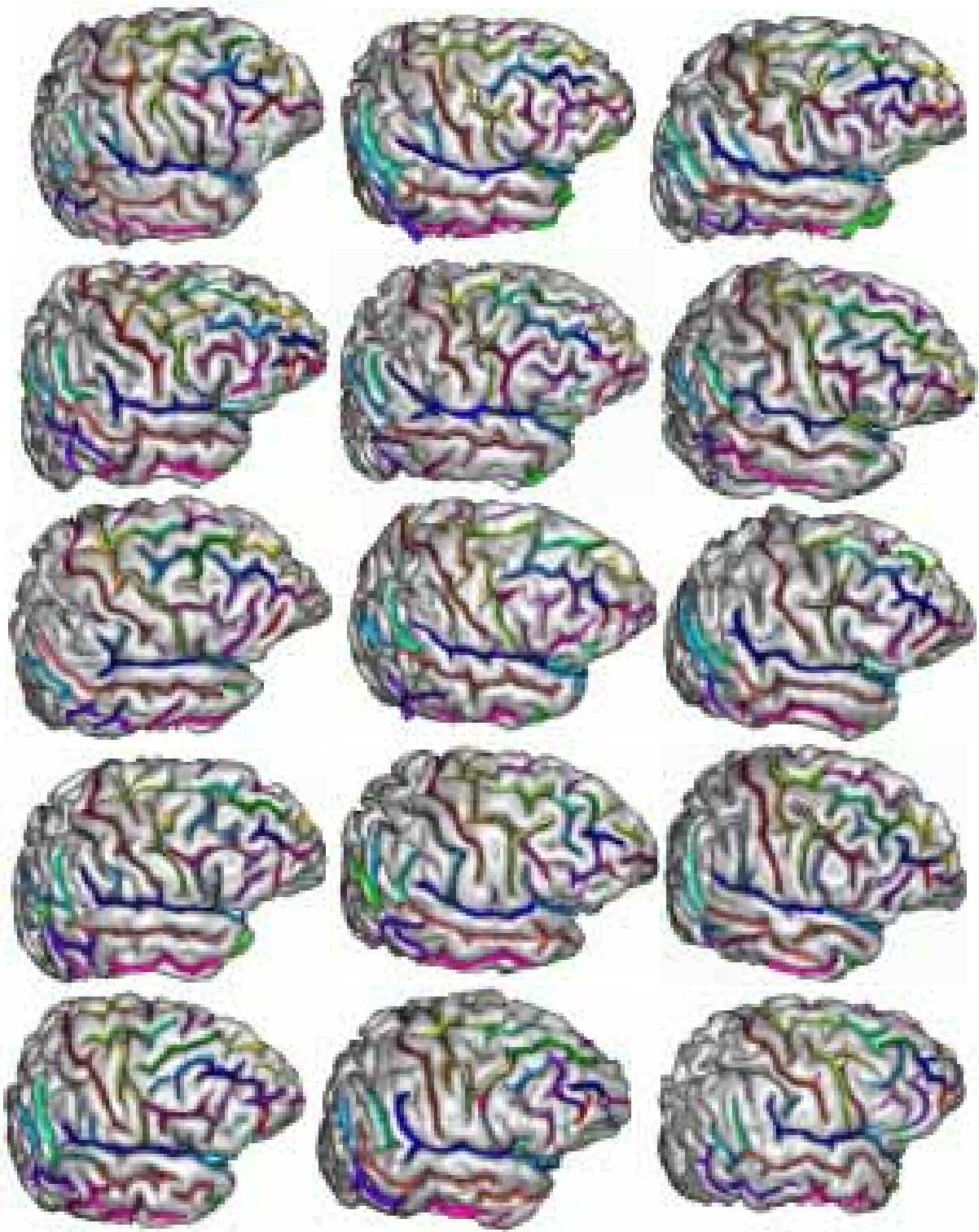

Fig. 1. A survey of the labelled database. The three first rows present nine brains of the learning base, the fourth row presents three brains of the test base, and the last row presents three brains of the generalization base. Each color labels one entity of the anatomical model. Several hues of the same color are used to depict different roots or stable branches of one given sulcus. For instance, color codes of main frontal sulci are: 2 reds $=$ central, 5 yellows $=$ precentral, 3 greens $=$ superior, 2 blues $=$ intermediate, 4 purples $=$ inferior, 8 blues Lateral fissure, red $=$ orbitary, rose $=$ marginal, yellow $=$ transverse... 


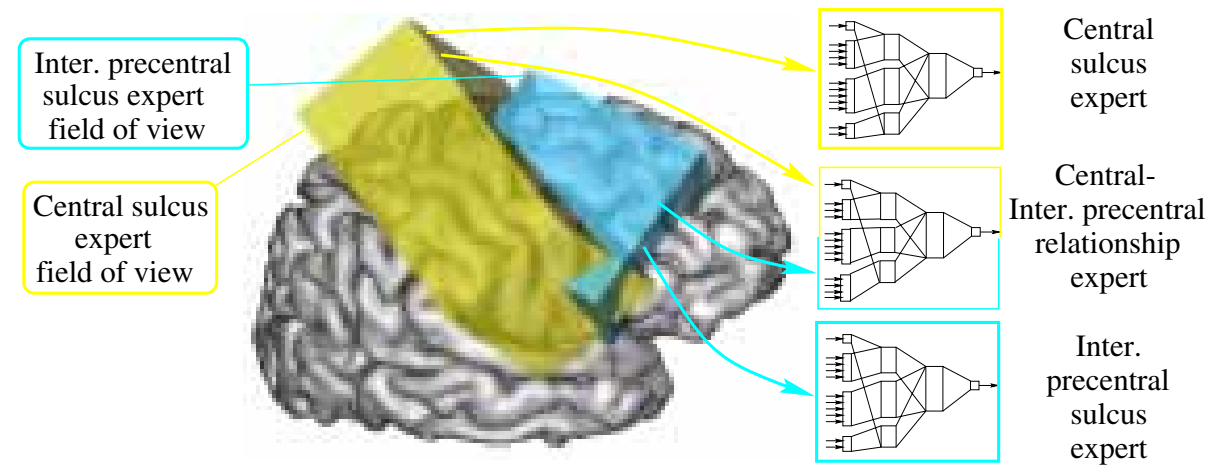

Fig. 2. 60 sulcus experts and 205 relationship experts are inferred from the learning base. Each expert evaluates the labelling of the nodes included in its field of view.

the model recently proposed by Lohman in [10]. This new type of anatomical model, however, requires further validations before being properly used by neuroscientists. Therefore, the results described in the following have been obtained after a conversion of this fine grain labelling to the standard nomenclature of Ono [17], which will allow comparisons to other group's works. This choice leads to a list 60 names for each hemisphere, where each name represent standard sulci and usual sulcus branches.

The 26 right hemispheres have been randomly separated into three bases: a learning base made up of 16 brains is used to train the local experts; a test base of 5 brains is used to stop the training before over-learning; and finally, a generalization base of 5 brains is used to assess the actual recognition performance of the system. We encourage the reader to study Fig. 1, which gives an idea of the variability of the folding patterns. Of course, our manual labelling can not be considered as a gold standard and could be questioned by other anatomists. It has to be noted, however, that a lot of information used to perform the manual recognition is concealed in the depth of the sulci.

\section{The Markovian Model}

Once a new brain has been virtually oriented according to a universal frame, for instance the Talairach system, the cortical area where one specific sulcus can be found is relatively small. This localization information can already lead to interesting recognition results $[8,9]$. Localization, however, is largely insufficient to perform a complete recognition. Indeed, a lot of discriminating power only stems from contextual information. This situation has led us to introduce a Markovian framework [15]. This framework provides us with a simple way of designing a probability distribution for the labelling: a Gibbs distribution relying on local potentials [6]. These potentials are inferred from the learning base. They embed interactions between the labels of neighboring nodes. These interactions 
are related to contextual constraints that must be adhered to in order to get anatomically plausible recognitions.

Two families of potential are designed. The first family evaluates the sulcus shapes and the second family evaluates the spatial relationships of pairs of neighboring sulci. Each potential depends only on the labels of a localized set of nodes, which corresponds to the Markov field interaction clique [6]. For a given graph, each clique corresponds to the set of nodes included in the field of view of the underlying expert (see Fig. 2). For sulcus experts, this field of view is defined from the learning base as a parallelepiped of the Talairach coordinate system. The parallelepiped is the bounding box of the sulcus instances in the learning base computed along the inertia axes of this instance set. For sulcus pair relationship experts, the field of view is simply the union of the fields of view of the two related sulcus experts. Pairs of sulci are endowed with an expert if at least $10 \%$ of the learning base brains possess an actual link between the two related sulci. For the model of the right hemisphere described in this paper, this rule leads to 205 relationship experts. The whole system, therefore, is made up of a congregation of 265 experts. Each expert potential is defined as the output of a standard multi-layer perceptron [22]. The expert single opinions are gathered by the Gibbs distribution, which gives the likelihood of a global labelling. Hence, the sulcus recognition amounts to minimizing the sum of all of the perceptron outputs.

\section{$5 \quad$ Expert Training}

Each expert is a multi-layer perceptron which is trained using the usual backpropagation algorithm [22]. Expert inputs are vectors of descriptors of the anatomical feature for which the expert is responsible. These descriptors constitute a compressed code of sulcus shapes and relationships. Sulcus shapes are summarized by 27 descriptors and sulcus relationships by 23 descriptors. These descriptors are computed from a small part of the graph corresponding to one single label (sulcus) or one pair of labels (relationship). A few Boolean logical descriptors are used to inform of the existence of a non-empty instance of some anatomical entity (sulcus, junction with the hemisphere hull, actual link between two sulci...). Continuous semantic descriptors are inferred from the semantic attributes [14] of the subgraph to be analyzed:

Sulcus expert: total size, minimal and maximal depth, gravity center localization, and mean orientation; length, mean direction, and extremity localization of the junction with the hemisphere hull;

Relationship expert: total sizes of both sulci; total sizes of each kind of links; minimal distance between the sulci; semantic attributes of the contact link (junction or burried gyrus [14]), namely junction localization, mean direction, distances between the contact point and the closest sulcus extremities, respective localization of the sulci, and angle between sulcus hull junctions. 

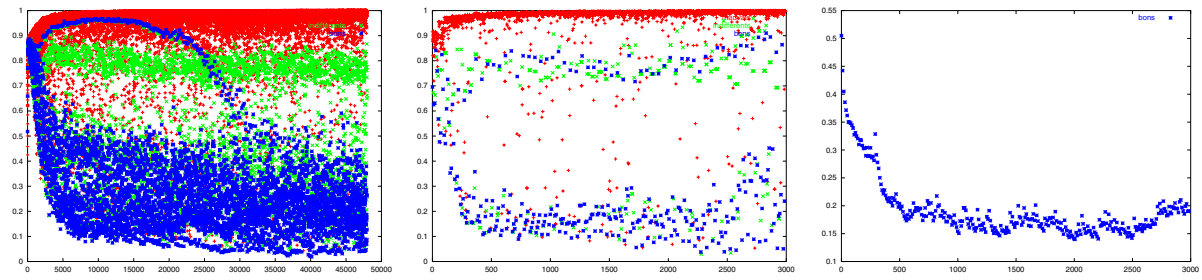

Fig. 3. A survey of the learning of the intermediate precentral sulcus expert. Dark points represent correct examples, light points ambiguous counterexamples, and middle grey points standard counter-examples. The first chart shows the evolution of the perceptron output for the learning base during the training. The second chart is related to the output for the test base. The third chart presents the evolution of the mean error on the test base. A consistent increase of this criterion corresponds to overlearning beginning.

Integer syntactic descriptors of the subgraph topology are also computed:

Sulcus expert: number of connected components using all links or only contact links; number of proximity links between contact related connected components, maximal gap between these components (continuous); number of internal links of "burried gyrus" type;

Relationship expert: numbers of contact related connected components of both sulci, numbers of such components implied in actual links between the sulci, number of contact points, number of links of "burried gyrus" type between the sulci, minimal depth of such links (continuous).

The perceptrons include two hidden layers and one output neuron. The first hidden layer is not fully connected to the input layer. Indeed, various experiments have led to the conclusion that a consistent packaging of the inputs lead to a better generalization power. Hence, the first hidden layer is split in several blocks feeded by a specific subset of inputs (see Fig. 2). For instance, syntactic descriptors feed one specific block.

The supervised learning of the experts relies on two kinds of examples. Correct examples extracted from the learning base must lead to the lowest output. Counter-examples are generated from correct examples through random modifications of some labels of the clique nodes. A continuous distance between the correct example and the generated counter-example is used to choose the taught output. Small distances lead to intermediate outputs while larger distances lead to the highest output. This balancing of the counter-example outputs was necessary to overcome learning difficulties fathered by ambiguous counterexamples.

The backpropagation algorithm is iteratively applied to the learning base using a one to ten ratio between correct examples and counter-examples. A stop criterion defined from the test base is used to avoid over-learning. This criterion relies on the sum of two mean errors computed respectively for correct examples and for counter-examples of the test base. The learning is stopped when this criterion presents a consistent increase or after a maximum number of iterations 


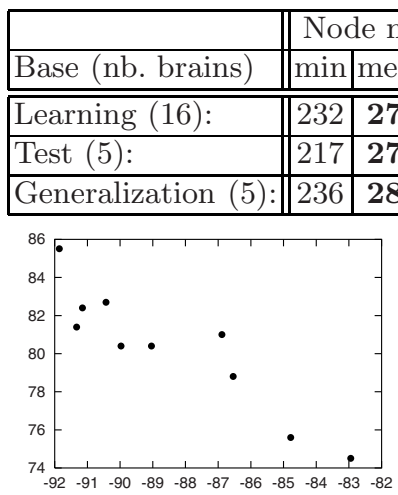

One brain of the learning base

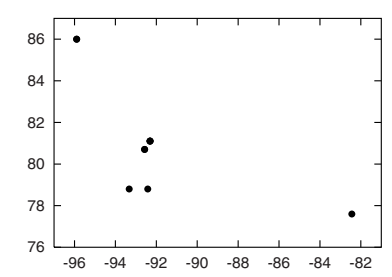

One brain of the test base

\begin{tabular}{|c|c|c||c|c|c|}
\multicolumn{3}{|c||}{$U_{\text {base }}$} & \multicolumn{3}{c|}{$U_{\text {annealing }}$} \\
\hline n & mean & max & min & mean & max \\
\hline 06 & $\mathbf{- 8 5}$ & -75 & -108 & $\mathbf{- 9 5}$ & -81 \\
\hline 8 & $\mathbf{- 4 6}$ & -24 & -96 & $\mathbf{- 8 5}$ & -74 \\
\hline 3 & $\mathbf{- 2 5}$ & -20 & -74 & $\mathbf{- 6 9}$ & -65 \\
\hline
\end{tabular}

Fig. 4. (top) Node number, recognition rate, energy of the manual labelling $\left(U_{\text {base }}\right)$, and energy of the automatic labelling for each base. (bottom) Recognition rate relative to final energy for ten different minimizations.

(cf. Fig. 3). The minimum value of this criterion is used to get a measure of confidence in the expert opinion. This measure is used to weight the output of this expert during the recognition process.

\section{Results}

The sulcus recognition process consists of the minimization of the energy made up of the weighted sum of the expert outputs. Each node label is chosen in a subset of the sulcus list corresponding to the expert fields of view which include this node. The minimization is performed using a stochastic algorithm inspired by the simulated annealing principle. This algorithm relies on a dedicated version of the Gibbs sampler [6]. For the following results, the minimization lasts about two hours on a conventional workstation. While an optimized implementation is planned in order to achieve a significant speed-up, it should be noted that the manual labelling work is even slower.

A global measure is proposed to assess the correct recognition rate. This measure corresponds to the proportion of cortical folds correctly identified according to the manual labelling. The contribution of each node to this global measure is weighted by its size (the number of voxels of the underlying skeleton [14]). The mean recognition rate on each of the three bases is proposed in Fig. 4. In order to check the reproducibility of the process, the minimization has been repeated ten times with different initializations for one brain of each base (see Fig. 4). This experiment has shown that the recognition rate is related to the depth of the local minimum obtained by the optimization process.

The recognition rate obtained for the generalization base is $76 \%$, which is very encouraging considering the variability of the folding patterns. As matters stand relative to our understanding of this variability, it should be noted that numerous "errors" of the system correspond in fact to ambiguous configurations. 
In fact, after a careful inspection of the results, the neuroanatomist of our team often admits to a preference for the automatic labelling. Moreover, the automatic system often corrects flagrant errors due to the cumbersome nature of the manual labelling. Increasing the size of the learning and test bases should help to improve the results. We plan also to develop a system using several experts for each anatomical entity in order to get a better management of the coding of the structural variability [20]. This work will include automatic adaptation of the topology of the neural networks to each expert.

\section{Conclusion}

A number of approaches relying on the deformable atlas paradigm consider that anatomical a priori knowledge can be completely embedded in iconic templates. While this point of view is very powerful for anatomical structures presenting low inter-individual variability, it seems insufficiently versatile to deal with the human cortical anatomy. This observation has led several teams to investigate approaches relying on higher levels of representation. All these approaches rely on a preprocessing stage which extracts sulcal related features describing the cortical topography. These features can be sulcal points [3], sulcal lines inferred from skeletons [21, 2], topologically simple surfaces [14], 2D parametric models of sulcal median axis $[7,24,25]$, crest lines $[5,11]$ or cortex depth maxima $[10,19]$. In our opinion, this direction of research can lead further than the usual deformable template approach. In fact these two types of work should be merged in the near future. It has to be understood, however, that some of the challenging issues about cortical anatomy mentioned in the introduction require new neuroscience results to be obtained. As such, image analysis teams addressing this kind of research must be responsible for providing neuroscientists with new tools in order to speed-up anatomical and brain mapping research. Our system is used today to question the current understanding of the variability and to help the emergence of better anatomical models. Various direct applications have been developed in the fields of epilepsy surgery planning and brain mapping.

\section{References}

[1] Ruzena Bajcsy and Chaim Broit. Matching of deformed images. In IEEE Pric. Sixth Int. Conf. on Pattern Recognition, pages 351-353, October 1982.

[2] A. Caunce and C. J. Taylor. Using local geometry to build 3D sulcal models. In IPMI'99, LNCS 1613, pages 196-209. Springer Verlag, 1999.

[3] H. Chui, J. Rambo, J. Duncan, R. Schultz, and A. Rangarajan. Registration of cortical anatomical structures via robust 3D point matching. In IPMI'99, LNCS 1613, pages 168-181. Springer Verlag, 1999.

[4] D. L. Collins, Le Goualher G., and A. C. Evans. Non-linear cerebral registration with sulcal constraints. In MICCAI'98, LNCS-1496, pages 974-984, 1998.

[5] J. Declerck, G. Subsol, J.-P. Thirion, and N. Ayache. Automatic retrieval of anatomical structures in 3D medical images. In CVRMed, LNCS-905, pages 153$162,1995$.

[6] S. Geman and D. Geman. Stochastic relaxation, gibbs distributions, and the bayesian restoration of images. IEEE PAMI, 6(6):721-741, 1984. 
[7] G. Le Goualher, C. Barillot, and Y. Bizais. Modeling cortical sulci using active ribbons. Int. J. Pattern Recognit. Artific. Intell., 11(8):1295-1315, 1997.

[8] G. Le Goualher, D. L. Collins, C. Barillot, and A. C. Evans. Automatic identification of cortical sulci using a 3D probabilistic atlas. In MICCAI'98, MIT, LNCS-1496, pages 509-518. Springer Verlag, 1998.

[9] G. Le Goualher, E. Procyk, D. L. Collins, R. Venugopal, C. Barillot, and A. C. Evans. Automated extraction and variability analysis of sulcal neuroanatomy. IEEE Medical Imaging, 18(3):206-217, 1999.

[10] G. Lohmann and Y von Cramon. Automatic detection and labelling of the human brain cortical folds in MR data sets. In ECCV, pages 369-381, 1998.

[11] A. Manceaux-Demiau, J.-F. Mangin, J. Regis, O. Pizzato, and V. Frouin. Differential features of cortical folds. In Springer-Verlag, editor, CVRMED/MRCAS, Grenoble, LNCS-1205, pages 439-448, 1997.

[12] J.-F. Mangin. Entropy minimization for automatic correction of intensity nonuniformity. In MMBIA, South Carolina, pages 162-169, 2000.

[13] J.-F. Mangin, O. Coulon, and V. Frouin. Robust brain segmentation using histogram scale-space analysis and mathematical morphology. In MICCAI'98, MIT, LNCS-1496, pages 1230-1241. Springer Verlag, 1998.

[14] J.-F. Mangin, V. Frouin, I. Bloch, J. Regis, and J. López-Krahe. From 3D MR images to structural representations of the cortex topography using topology preserving deformations. J. Mathematical Imaging and Vision, 5(4):297-318, 1995.

[15] J.-F. Mangin, J. Regis, I. Bloch, V. Frouin, Y. Samson, and J. Lopez-Krahe. A Markovian random field based random graph modelling the human cortical topography. In CVRMed, Nice, LNCS-905, pages 177-183. Springer-Verlag, 1995.

[16] J.-F. Mangin, J. Régis, and V. Frouin. Shape bottlenecks and conservative flow systems. In MMBIA, San Francisco, pages 319-328, 1996.

[17] M. Ono, S. Kubik, and C. D. Abernethey. Atlas of the Cerebral Sulci. Georg Thieme Verlag, 1990.

[18] J. Régis, J.-F. Mangin, V. Frouin, F. Sastre, J. C. Peragut, and Y. Samson. Generic model for the localization of the cerebral cortex and preoperative multimodal integration in epilepsy surgery. Stereotactic Functional Neurosurgery, 65:72-80, 1995.

[19] M. E. Rettmann, C. Xu, D. L. Pham, and J. L. Prince. Automated segmentation of sulcal regions. In MICCAI'99, Cambridge, UK, LNCS-1679, Springer-Verlag, pages 158-167, 1999.

[20] D. Rivière, J.-F. Mangin, J.-M. Martinez, F. Chavand, and V. Frouin. Neural network based learning of local compatibilities for segment grouping. In SSPR'98, LNCS-1451, pages 349-358. Springer Verlag, 1998.

[21] N. Royackkers, M. Desvignes, H. Fawal, and M. Revenu. Detection and statistical analysis of human cortical sulci. NeuroImage, 10:625-641, 1999.

$[22]$ D. E. Rumelhart, G. E. Hinton, and R. J. Williams. Learning internal representations by error backpropagation, pages 318-362. MIT Press, 1986.

[23] P. M. Thompson, R. P. Woods, M. S. Mega, and A. W. Toga. Mathematical/computational challenges in creating deformable and probabilistic atlases of the human brain. Human Brain Mapping, 9:81-92, 2000.

[24] M. Vaillant and C. Davatzikos. Finding parametric representations of the cortical sulci using active contour model. Medical Image Analysis, 1(4):295-315, 1997.

[25] X. Zeng, L. H. Staib, R. T. Schultz, H. Tagare, L. Win, and J. S. Duncan. A new approach to 3D sulcal ribbon finding from MR images. In MICCAI'99, Cambridge, UK, LNCS-1679, Springer-Verlag, pages 148-157, 1999. 